\title{
New challenges in electrochemistry and electroanalysis
}

\author{
Allen J. Bard \\ Department of Chemistry and Biochemistry, The University of Texas at Austin, \\ Austin, Texas 78712
}

\begin{abstract}
The challenges and opportunities that face electrochemistry and electroanalytical chemistry arise from societal needs, advances in technology, and from the emergence of important new scientific areas and problems. Our ability to construct very small electrodes, sometimes with nanometer dimensions, and to measure small currents generated at these, have made possible measurements at very short times and in very resistive solvents. These new solvents, such as liquid $\mathrm{SO}_{2}$ or supercritical fluids, have, in turn, led to the investigation of greatly expanded potential regimes. The invention of scanning probe microscopies (e.g., STM, SECM) now allow very high resolution examination of the topography and chemical nature of electrode surfaces. Electrochemical methods should continue to play an important role in new fields, e.g., molecular biology and materials science. For example, interactions of molecules with DNA can be probed by voltammetric measurements and highly sensitive analytical methods based on electrogenerated chemiluminescent labels attached to antibodies have been developed.
\end{abstract}

\section{INTRODUCTION}

New challenges arise in scientific fields from "pushes and pulls" (ref. 1). The pull comes from the desire to address societal needs (Fig. 1) and the push from the advances in science and technology (Fig. 2). As shown in Fig. 1, electrochemistry and electroanalysis can contribute to many areas of concern to society. For example, in health care, sensors will continue to be an important area; enzyme electrodes are already finding use in the monitoring of glucose levels in blood. Sensors that could be used in vivo over long periods of time and sensitive analytical methods that could be used for antibodies, antigens, and DNA would be especially valuable. We will return to this topic in this lecture. Electrochemistry has always played an important role in the energy area. Solar energy conversion through photoelectrochemistry at semiconductors remains an active area. Large scale utilization of fuel cells and batteries in electric vehicles is on the horizon. Society's environmental concerns can be helped by solar energy sources and systems that are cleaner than those based on the conventional combustion of fossil fuels. Electrochemical and photoelectrochemical methods can also be used to treat wastes and purify water. Finally, there are national concerns about security and economic competitiveness. New areas like nanotechnology, biotechnology, and advanced materials are emerging, and, as discussed below, electrochemistry can contribute here as well.

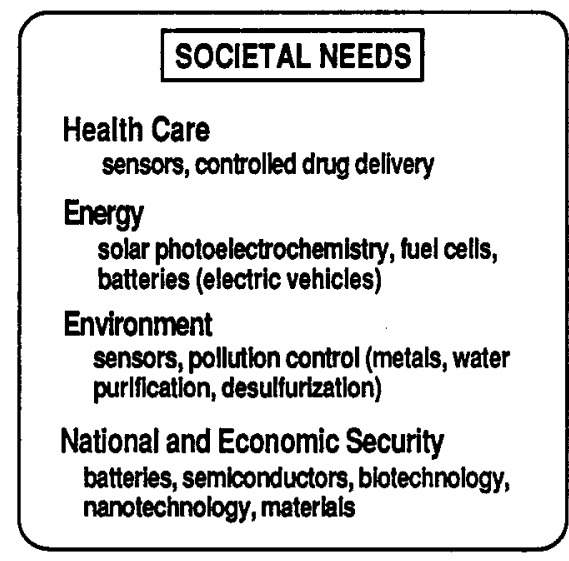

Fig. 1

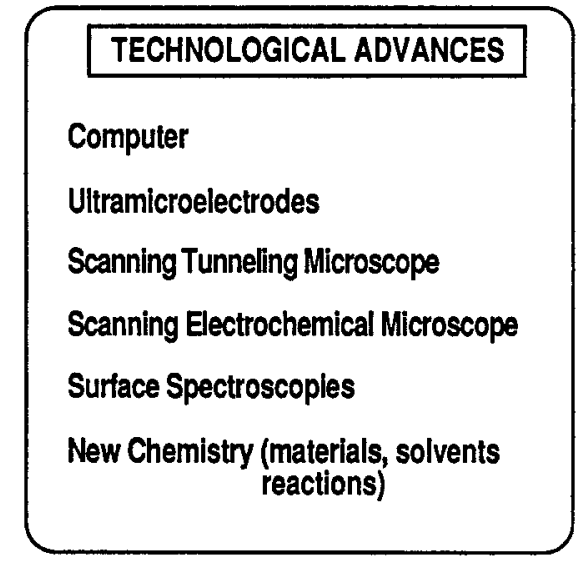

Fig. 2 
The push arises when new tools and instruments become available for studying electrochemical systems and new areas of science emerge that can be studied by electrochemical methods. For example, the availability of different types of surface spectroscopies (XPS, AES, LEED...) and scanning probe microscopies, such as scanning tunneling microscopy (STM) and atomic force microscopy (AFM), have provided new insights into surface structure and surface processes. Atomic level images of electrode surfaces in situ are already possible under favorable conditions, as discussed below. New materials, such as electronically conducting polymers and the fullerenes, new solvents, and the ability to carry out investigations under extreme conditions (e.g., in supercritical fluids) will also push to broaden the horizons of electrochemistry.

\section{EXPANDING THE LIMITS}

Electrochemical methods operate within limits of time, space, potential, etc., that are often defined by the available instrumentation and materials (Fig. 3). We are challenged to expand these limits for electrochemical methods-to study electrode reactions with higher spatial resolution, at shorter times, at more extreme potentials, at higher pressures or at lower temperatures, and explore the new science that can be found in these regimes.

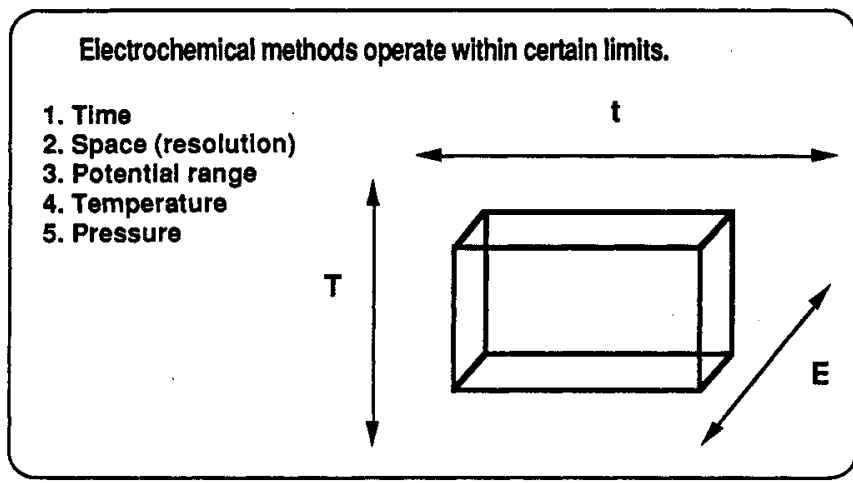

Fig. 3

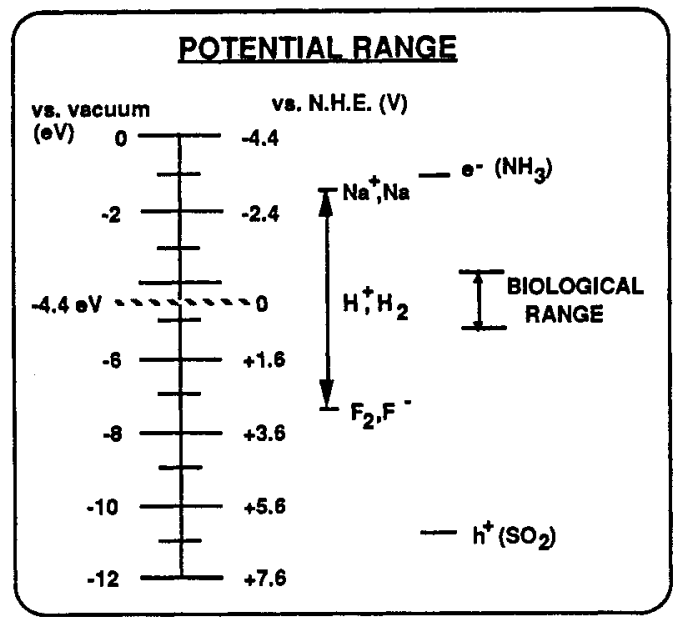

Fig. 4

\section{Potential}

The electron transfer (et) processes that we can study depend upon the limits of potential that are available with a given solvent-electrolyte-electrode system (Fig. 4). We might recall that it was the implementation of electrochemistry in synthesis, e.g., by Davy and Faraday, that led to the production of substances like sodium and potassium that could not be obtained with available, naturally-occurring, reactants. The introduction of aprotic solvents in electrochemistry similarly allowed new reactions of organic and inorganic compounds to be explored and intermediates like radical ions to be studied. What are the limits of potentials for reduction and oxidation reactions?

The most negative limit for cathodic reactions in solution is probably near that of the electron in vacuum, $e^{-}$vac (about $-4.4 \mathrm{~V}$ vs N.H.E.). (Certain solvents, like liquid $\mathrm{He}$, might allow significantly more negative potentials, but are not practical for electrochemical studies.) Solvated electrons already can be electrogenerated in solvents like liquid ammonia and the amines at potentials that differ from $e^{-} \mathrm{vac}$ by only the free energy of solvation (ref. 2 ). Thus we are probably quite close to the practical negative limit.

The positive limit for anodic reactions is governed by the oxidative stability of the electrode material, the ease of oxidation of the electrolyte, and the potential for the production of a solvated hole $(\mathrm{h}+\mathrm{solv})$ in the solvent of interest, which is near that of the solvent cation radical. There has been continuous progress in the use of solvents that allow ever more positive potentials to be applied before the solvent limit. The introduction of microelectrodes has allowed the use of solvents that would be too resistive for studies with larger electrodes, because the smaller currents that flow decrease contributions from iR-drop in the potential measurements (ref. 3). They also permit studies in the presence of low concentrations of supporting electrolyte. For example, liquid $\mathrm{SO}_{2}$, with the appropriate supporting electrolyte (such as $\mathrm{CsAsF}_{6}$ ), can be used out to almost $+6 \vee v s$ S.C.E. Even aliphatic hydrocarbons and quaternary ammonium lons can be oxidized in this solvent (Fig. 5) (ref. 4). Further extensions to even more positive potentials appear possible and await new solventelectrolyte systems. 

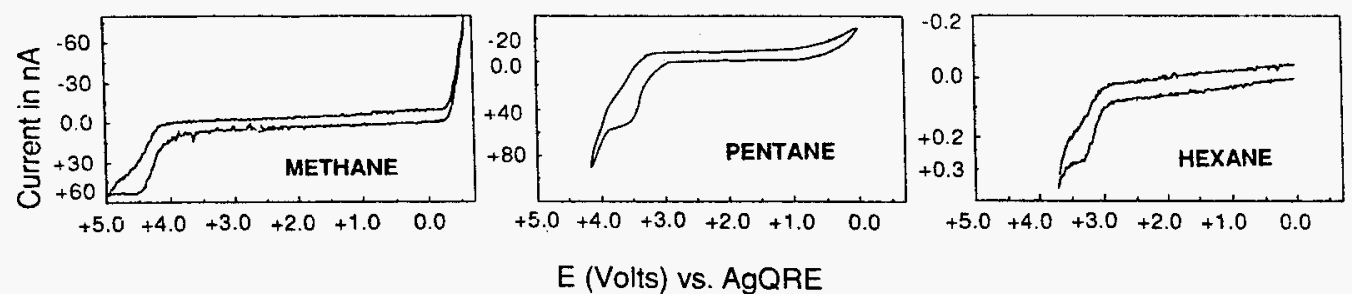

Figure 5. Cyclic voltammograms of $n$-hydrocarbons at $-70^{\circ} \mathrm{C}$ in liquid $\mathrm{SO}_{2}$. Supporting electrolyte used: for: $1 \mathrm{~atm}$ methane, $30 \mathrm{mM} \mathrm{CsAsF} 6(\mathrm{~V}=5 \mathrm{~V} / \mathrm{s})$; for $50 \mathrm{mM}$ pentane, $0.2 \mathrm{M} \mathrm{TBAAsF}_{6}(\mathrm{v}=100 \mathrm{~V} / \mathrm{s})$; for $10 \mathrm{mM}$ hexane, $0.2 \mathrm{M} \mathrm{TBAAsF}_{6}(\mathrm{~V}=10 \mathrm{~V} / \mathrm{s})$.

(From ref. 4.)

\section{Time}

About 40 years ago electrochemical methods were among those that could be used to study "fast" reactions (i.e., in the millisecond regime). However, the invention of the laser, and associated fast spectroscopic instrumentation, has moved time measurements in chemistry into the picosecond and femtosecond regions, leaving electrochemical measurements far behind. Nevertheless, interesting et reactions remain to be explored in the microsecond and nanosecond regions and there are signs that electrochemical measurements within this time scale are possible. Again, very small microelectrodes, with minimized contributions from iR and double layer charging effects, have been shown to be useful in fast electrochemical measurements. Cyclic voltammetry at scan rates up to $10^{6} \mathrm{~V} / \mathrm{s}$ (equivalent to a time window of $26 \mathrm{~ns}$ ) have been reported (refs. 5,6). Similarly, sub-microsecond potential step experiments have been carried out. For example, Xu and Faulkner have constructed a cell and instrumentation in which double layer charging times of 30-50 ns are observed (ref. 7). Many of the reactions that have been studied by electrochemical methods over the years can now be reinvestigated in these short time regimes with the observation of intermediates not previously detectable.

\section{Space}

Over the past years there has been a growing emphasis on the spatial characterization of surfaces along with a new ability to examine surfaces with very high resolution. For example, the scanning tunneling microscope (STM) can be used with conducting and semiconducting substrates to obtain information about surface topography on the nanometer, and in favorable cases, the atomic ( $A$ ) level (ref. 8). Thus, a new tool is available to study electrode surfaces and electrochemical processes like electrodeposition and corrosion (ref. 9). As an example, let me describe some recent work in our laboratory on the deposition of $\mathrm{Pb}$ on highly oriented pyrolytic graphite (HOPG) (ref. 10). HOPG can be cleaved to form a fresh atomically smooth surface. We recently showed that by heating HOPG in air at $650^{\circ} \mathrm{C}$ pits with dimensions in the $10-100 \mathrm{~nm}$ range can be prepared (Fig. 6) (ref. 11). These pits serve as useful markers for repeated STM scans during a study of a surface process and also allow one to distinguish processes that occur on the HOPG basal plane and edge sites around the pit. The deposition of lead on HOPG during a potential step and its subsequent stripping are shown in Fig. 7 (ref. 10). Note the formation (or migration) of metallic $\mathrm{Pb}$ to the pit edges during the deposition and its stripping during the oxidation step. Interestingly, over many experiments like this, we find that not all of the $\mathrm{Pb}$ is stripped from the surface during oxidation, perhaps because some particles lose electrical contact with the surface. Moreover, the locations of the $\mathrm{Pb}$ deposits indicate that there is some interaction of them with the scanning tip.

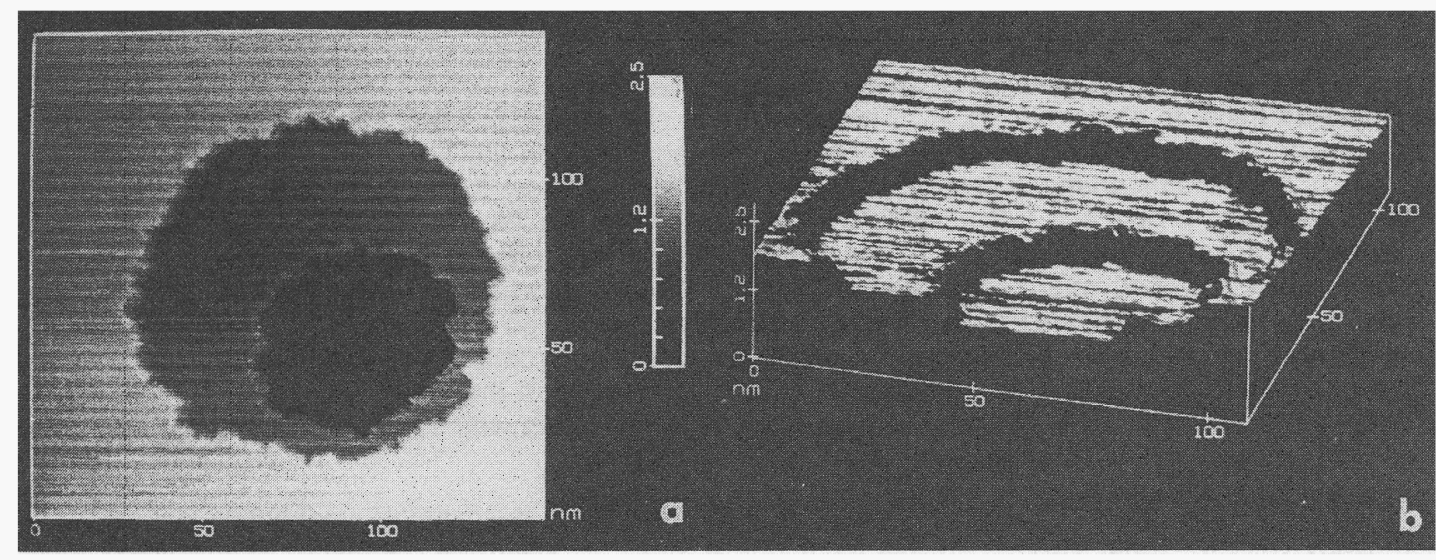

Fig. 6. STM image of a double etch pit formed on HOPG. The double pit is formed when a sample with an existing etch pit is heated at $650^{\circ} \mathrm{C}$ : (a) topographic view; (b) surface view. (From ref. 11.) 


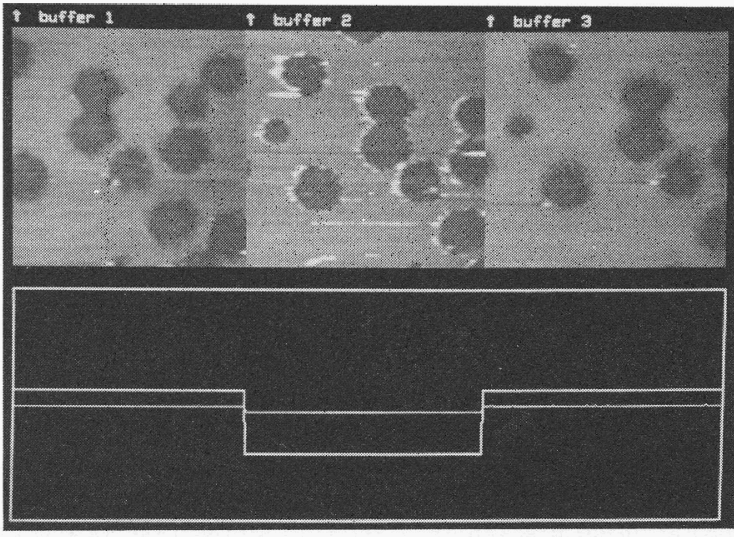

Fig. 7. In situ STM images showing the deposition and stripping of $\mathrm{Pb}$ on a single area of HOPG. The substrate potential is shown below the images. (1) The sample held at $+0.18 \mathrm{~V}$ vs SCE. (2) Deposition at -0.58 V v SCE. (3) Stripping at +0.18 V v SCE. The tip was ramped from 382 to $-400 \mathrm{mV}$ and back.

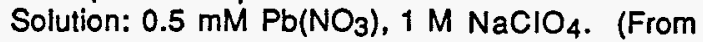
ref. 10.)

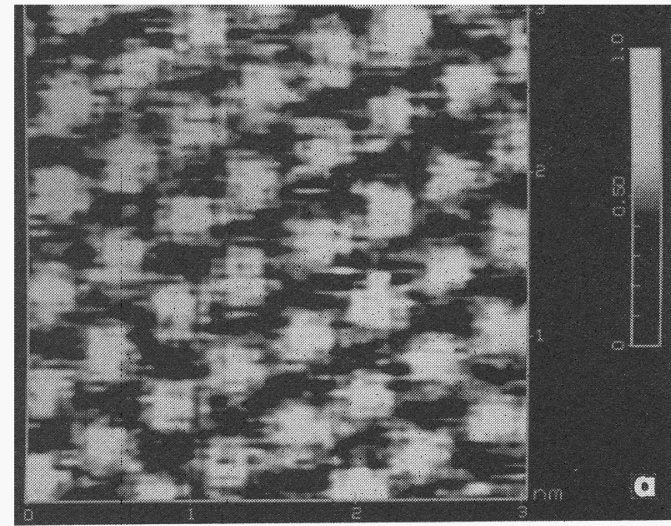

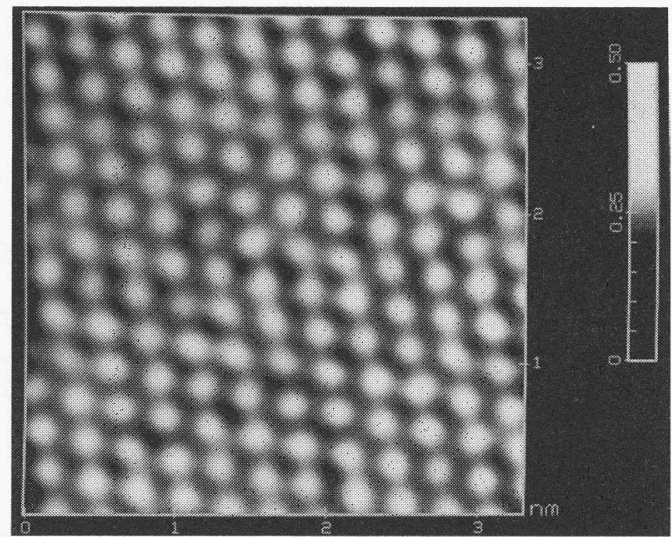

Fig. 8. STM image of Au (111) film evaporated on mica. Imaged in air, with tungsten tip biased at $20 \mathrm{mV}$; tunneling current $=3.0 \mathrm{nA}$. (From ref: 13.)

Fig. 9. (a) lodide coated Au (111), prepared by dosing the gold substrate in $10^{-2} \mathrm{M} \mathrm{KI}$ for $30 \mathrm{~min}$. Tip was biased at $50 \mathrm{mV}$; tunneling current $=1 \mathrm{nA}$. (From ref. 13.) (b) Adlattice structure of 4-aminothiophene (4-ATP) on Au (111). The 4-ATP was adsorbed onto the Au film by immersion in a $10 \mathrm{mM}$ solution of 4-ATP in EtOH for $24 \mathrm{~h}$. Tip was biased at $-31.1 . \mathrm{mV}$; tunneling current $=6.2 \mathrm{nA}$. Inset: Schematic of $(\sqrt{3} \times \sqrt{3}) \mathrm{R} 30^{\circ}$ adlattice on $\mathrm{Au}$ (111). (From ref. 14.)

An example where atomic resolution of an electrode surface can be seen, an STM scan in air of Au deposited on mica, is shown in Fig. 8. The structure is clearly that of Au (111) (refs. 12, 13). One can then examine the nature of various substances that are adsorbed on the Au surface, like iodine (Fig. 9a) (ref. 13) or 4-aminothiophene (Fig. 9b) (ref. 14). The results show that these molecules adsorb in three-fold sites to produce a $(\sqrt{3} \times \sqrt{3}) \mathrm{R} 30^{\circ}$ structure (inset, Fig. 9b). Similar results have been found for the adsorption of iodine on Pt (111) (ref. 15) and long chain thiols on Au (ref. 16). Clearly we are reaching the point where adsorption and surface processes on electrodes can be studied with molecular resolution and almost on an atom-by-atom basis. Although STM cannot be used with insulating substrates, atomic force microscopy (AFM) shows considerable promise for such studies (ref. 17). Both STM and AFM suffer from problems of sizeable interactions between the tip and surface, sometimes causing perturbations of the surface during the scanning process, as well as a general lack of chemical information in the resulting responses.

An alternative technique that is being developed in our laboratory is scanning electrochemical microscopy (SECM) (refs. 18,19). In this technique a small tip electrode, behaving as the working electrode in an electrochemical cell, is scanned above a substrate immersed in an electrolyte solution containing a redox couple (e.g., O/R). The presence of the substrate affects the faradaic current flowing 

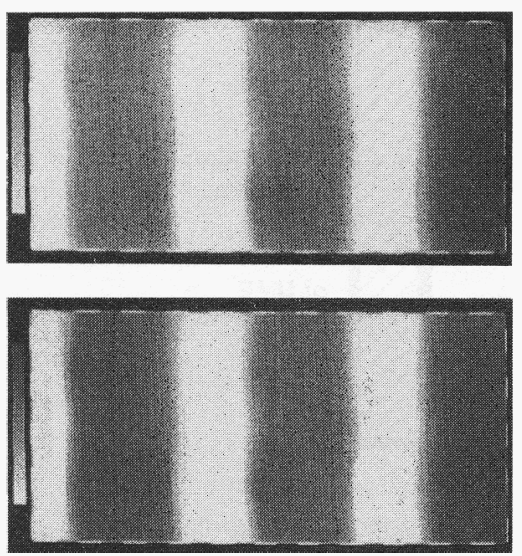

Fig. 10. SECM images of an interdigitated array of $\mathrm{Pt}$ and $\mathrm{SiO}_{2}$. Solution used: $40 \mathrm{mM} \mathrm{MVCl}_{2}, 2 \mathrm{M}$ $\mathrm{KCl}$. A Pt microdisk electrode (tip diameter $=$ $0.2 \mu \mathrm{m}$ ) was used. The tip potential was held at $-0.78 \mathrm{~V}$ vs SCE (where reduction of $\mathrm{MV}^{2}+$ to $M V+$ occurs). Light shades represent maximum it ( $\mathrm{Pt}$ bands) and dark shades minimum iT $\left(\mathrm{SiO}_{2}\right)$. The scale markings around the edge denote a $1-\mu \mathrm{m}$ distance. Upper image is from minimum to maximum current and lower image from average to maximum current. (From ref. 20.)

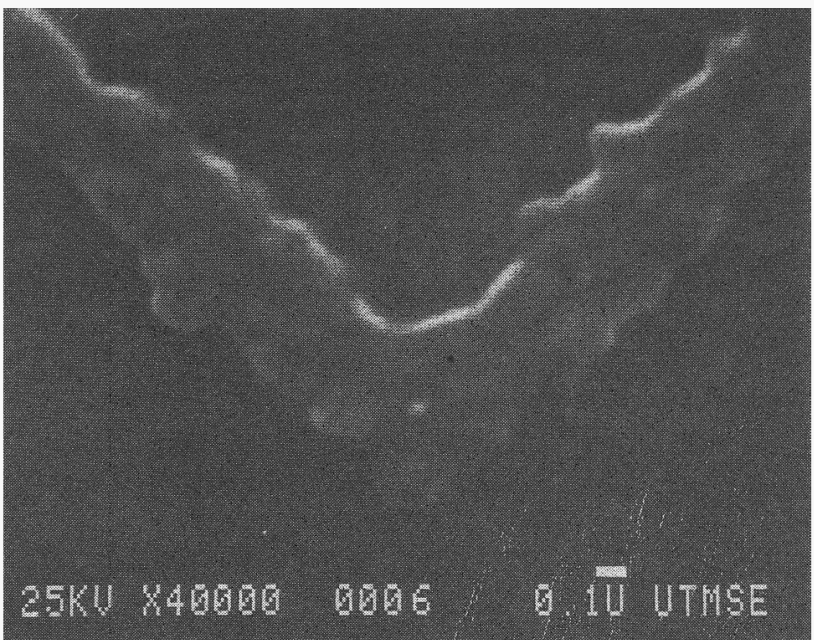

Fig. 11. SEM of a pattern of silver lines deposited by SECM in a Nafion film. The tungsten tip was biased at $5 \mathrm{~V}$ (tunneling current $=-0.5 \mathrm{nA}$, scan rate $=900 \AA / \mathrm{s})$. (From ref. 21.)

at the tip electrode, when the tip is close (i.e., within a few tip radii) to the substrate surface. Consider the case where the solution contains species $O$ and the tip is held at a potential where the reduction of $O$ to $R$ occurs. When the substrate is a conductor and the $R$ generated at the tip can be oxidized back to $O$, the current at the tip will be larger than that when it is far from the substrate (positive feedback to tip). When the substrate is an insulator, it partially blocks diffusion of $O$ to the tip, and the current is decreased (negative feedback to the tip). Thus the magnitude of the current observed as the tip is scanned near the substrate surface is related to surface topography and also the rate of oxidation of species $O$ at the surface. Typical SECM images for a scan over an interdigitated array consisting of Pt bands separated by silica spaces are shown in Fig. 10 (ref. 20). By suitable choice of the couple O/R, the chemical nature of the surface can be probed. Moreover the tip current-distance response can be related quantitatively to theoretical models with high accuracy. SECM can also be used to probe the kinetics of reactions at the substrate surface (and, hence, image differential reactivity on surfaces) and in the solution gap between tip and substrate. Because faradaic currents flow at the tip in SECM, modifications of surfaces via electrodeposition and etching can be carried out with high resolution. An example of a line of $\mathrm{Ag}$ electroplated in a polymer (Nafion) film by SECM is shown in Fig. 11 (ref. 21). Electrochemistry should play a useful role in nanotechnology, i.e., in the construction and characterization of systems with high resolution.

\section{Quantity}

The search for methods that can analyze for smaller and smaller quantities of a substance continues and electroanalytical methods should prove useful in this field as well. Such methods are needed, for example, to find very low concentrations or very small amounts of species (e.g., antibodies, antigens, DNA) in biological systems or on surfaces. Since electrochemical methods can readily detect fractions of monolayers of materials (where a monolayer represents about $10^{-10}$ moles $\mathrm{cm}^{-2}$ ), one should be able to determine very small quantities of material localized on the surface of a microelectrode. For example, if a material could be adsorbed or trapped on the surface of an electrode of $10 \mu \mathrm{m}$ diameter, measurement of quantities in the $4 \times 10^{-18}$ mol (2.4 million molecule) range should be possible, but challenging! Detection limits can be extended by amplification methods, e.g., by using enzymes or electrogenerated chemiluminescence (ECL). For example, in an electrochemical enzyme immunoassay method, $4.6 \times$ $10^{-21}$ moles of $\operatorname{lgG}$ can be determined (ref. 22). The SECM can also be used for the analysis of species on small portions of a surface. For example, a 3-30 $\mu \mathrm{L}$ drop of solution can be placed on a surface and SECM used to analyze for changes in the contents of the drop caused by adsorption/desorption or dissolution reactions (Fig. 12) (ref. 23). Electrochemical detection methods are also being used with electrodes small enough to sample single cells (ref. 24). 


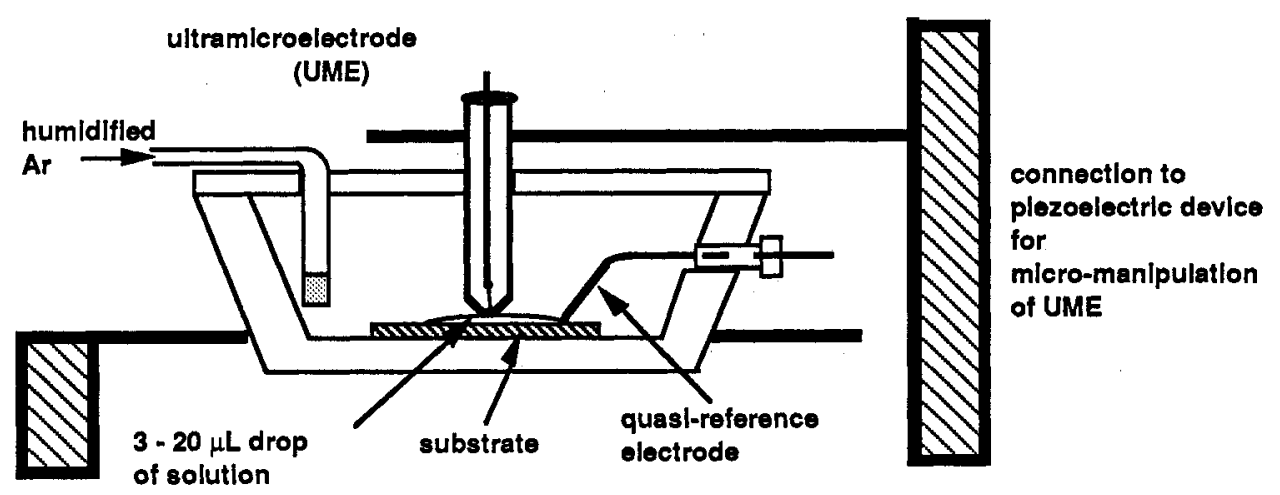

Fig. 12. Schematic of the experimental apparatus for adsorption studies via UME voltammetry in a drop of solution. (From ref. 23.)

\section{Temperature and pressure}

By extending the working range for electrochemical studies to very low temperatures it might be possible to probe the behavior of superconductive materials as electrodes. The challenge is to find media that are remain liquid at the low temperatures while still maintaining a reasonable conductivity. For example, electrochemistry in liquid nitrogen seems unlikely. However, other solvents are being explored in the same temperature region (ref. 25). Higher temperatures and pressures are also of interest. The properties of solvents (e.g., dielectric constant and viscosity) are critically dependent upon the temperature and pressure range in the near critical and supercritical region. The dielectric constant of water decreases substantially, and water becomes infinitely miscible with benzene, beyond the critical point ( $374^{\circ} \mathrm{C}$ and $221 \mathrm{bar}$ ). Moreover diffusion coefficients and rate constants can become very high in this region. Electrochemical studies in supercritical water and other solvents $\left(\mathrm{NH}_{3}, \mathrm{MeCN}, \mathrm{SO}_{2}\right)$ have already been reported (ref. 26), but much remains to be done. Similarly, one looks to studies at extremely high pressure at lower temperatures and the discovery of new molten salt systems. The experimental challenges in these areas are high, but enough has been reported already to demonstrate that these regions can be explored electrochemically.

\section{APPLICATIONS TO MOLECULAR BIOLOGICAL PROBLEMS}

Molecular biology remains a frontier field in science and electrochemistry and electroanalysis can play a role in its development. I briefly discuss here two recent applications from our laboratory-the use of electrochemical methods to study the interactions of molecules with DNA and immunological analysis based on electrogenerated chemiluminescence.

\section{Metal chelate-DNA interactions}

Many small molecules will interact with DNA. These interactions are important because they can affect DNA replication and can also form the basis of reactions that can cleave DNA and so play a role in obtaining the base sequence in DNA and understanding DNA structure. These interactions are usually studied by spectroscopic or dialysis methods. There are several modes of interaction: intercalation between the DNA bases, binding in the minor groove, and electrostatic interactions with the negatively charged DNA backbone. For example a number of metal chelates, e.g., Co(phen) $3^{3+}$ (where phen is 1,10 phenanthroline), will intercalate with DNA. Electrochemical studies can be used to measure the strength of binding of the complex and the size of the binding site (i.e., number of base pairs) by studying the voltammetry of the complex in the absence and presence of DNA and noting shifts in standard potential caused by the interaction (ref. 27). One can also measure the change in the diffusion current for metal chelate electrochemistry caused by the large decrease in diffusion coefficient $(D)$ when the complex is bound to DNA. A similar strategy can be employed with the Mn ${ }^{\prime l l}$ complex of meso( $N$-methyl-4pyridiniumyl)porphine (MnIII TMPyP), which binds in the minor groove of DNA and, under proper conditions, promotes DNA strand cleavage (ref. 28). The effect of DNA on the differential pulse voltammetric (DPV) wave of Mn 111 TMPyP is shown in Fig. 13. The large decrease in the height of the wave is caused by the large decrease in D for Mn III TMPyP upon binding to DNA. The binding constant and binding site size can be obtained by titrating the complex with DNA and noting the decrease in the peak current (Fig. 14). Electrochemical reduction of $\mathrm{Mn}$ III TMPyP in the presence of oxygen caused DNA cleavage, probably via formation of reduced oxygen species from the reaction between $\mathrm{O}_{2}$ and the $\mathrm{Mn}^{\prime \prime}$ complex. Electrochemical studies of other types of molecules, especially those that can recognize specific base sequences, e.g., oligonucleotides containing tethered electroactive groups, would be of special interest. 

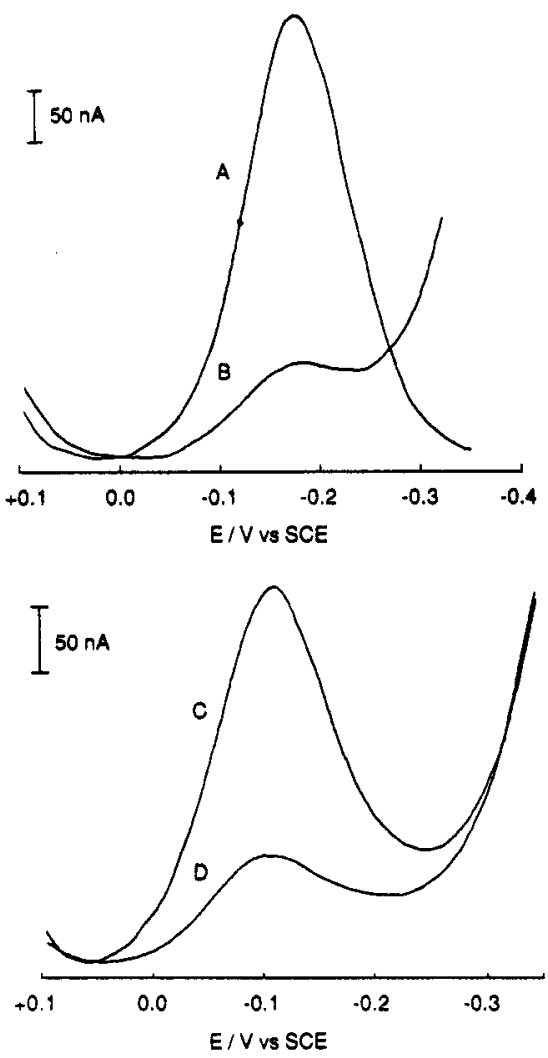

Fig. 13. Differential pulse voltammograms of $(A, B), 0.1 \mathrm{mM} M n^{I I I} T M P y P$ and $(C, D), 0.1 \mathrm{mM} F e^{\text {Ill }}$ TMPyP, $(A, C)$ in the absence of DNA and in the presence of sonnicated CT DNA with (B) $0.8 \mathrm{mM}$ NP, (D) $4,8 \mathrm{mM}$ MP. (From ref. 28.)
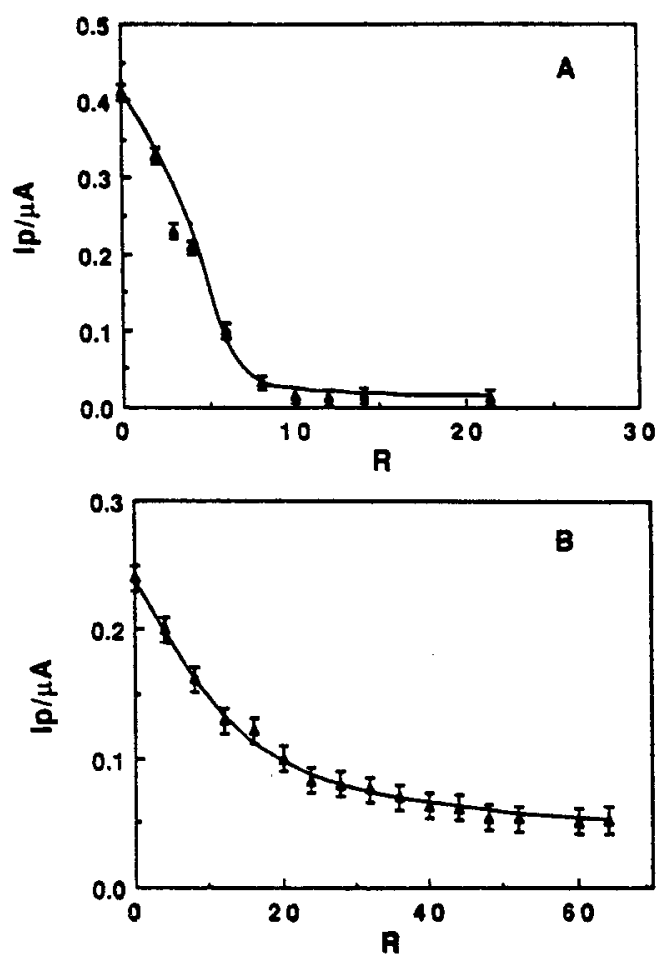

Fig. 14. Titration curve of (A) $0.1 \mathrm{mM}$ Mn'IITMPyP and (B) $0.1 \mathrm{mM}$ FelliTMPyP with sonnicated CT DNA. Points represent the experimental dat and solid curves represent the best fit obtained assuming mobile equilibria. Error bars correspond to $\pm 2 \sigma$ for five measurements. (From ref. 28.)

Similar strategies could be used to extend this approach to other systems. Nonelectroactive DNA binders can probably be studied by their competitive interactions with electroactive molecules. Studies of the binding and cleavage of other biologically-interesting molecules, such as RNA, peptides, and proteins should also be possible. Clearly electrochemical methods can complement spectroscopic and other approaches to studies of bioconjugation.

\section{ECL immunological analysis}

Very sensitive analytical methods can be devised based on electrogenerated chemiluminescence (ECL). ECL is the production of light-emitting species through redox reactions of reactants generated at an electrode surface (ref. 29). Many ECL reactions have been discovered, and ECL as an analytical method offers the advantages of very high sensitivity and selectivity. For example, because light is produced without excitation radiation, fluorescence of impurities and scattered light is not a problem. A widelyinvestigated ECL reaction involves the oxidation of $R u(b p y) 3^{2+}$ at an electrode in the presence of oxalate or tri(n-propyl)amine (refs. 30,31 ). The reaction of electrogenerated $R u(b p y) 3^{3+}$ with oxalate leads to the excited state of $R u(b p y) 3^{2+}$ by the following reactions:

$$
\begin{aligned}
\mathrm{Ru}(\text { bpy })_{3}^{3+}+\mathrm{C}_{2} \mathrm{O}_{4}{ }^{2-} & \rightarrow \mathrm{Ru}(\text { bpy })_{3}{ }^{2+}+\mathrm{C}_{2} \mathrm{O}_{4}^{-} \\
\mathrm{C}_{2} \mathrm{O}_{4}^{-} & \rightarrow \mathrm{CO}_{2}+\mathrm{CO}_{2}^{-} \\
\mathrm{Ru}(\mathrm{bpy})_{3}{ }^{3+}+\mathrm{CO}_{2}^{-} & \rightarrow \mathrm{Ru}(\mathrm{bpy})_{3}{ }^{2+*}+\mathrm{CO}_{2}
\end{aligned}
$$

Measurement of the emission from the excited state, Ru(bpy) $3^{2+*}$, can be used to determine very low concentrations (e.g., $10^{-13} \mathrm{M}$ ) of $R u\left(\right.$ bpy) $3^{2+}$ (ref. 32 ). If this molecule is used as a label on a biological molecule, such as an antigen or antibody, then it can form the basis of a sensitive bioassay method. Such methods are currently under development in a flow injection analysis apparatus that is totally automated and that can be used for different determinations based on a Ru(bpy) $3^{2+}$ label (refs. 33,34). The principles of immunoassays based on this method are given in Fig. 15. The use of other labels which emit at different wavelengths would make possible multiple analyses on a given sample. 


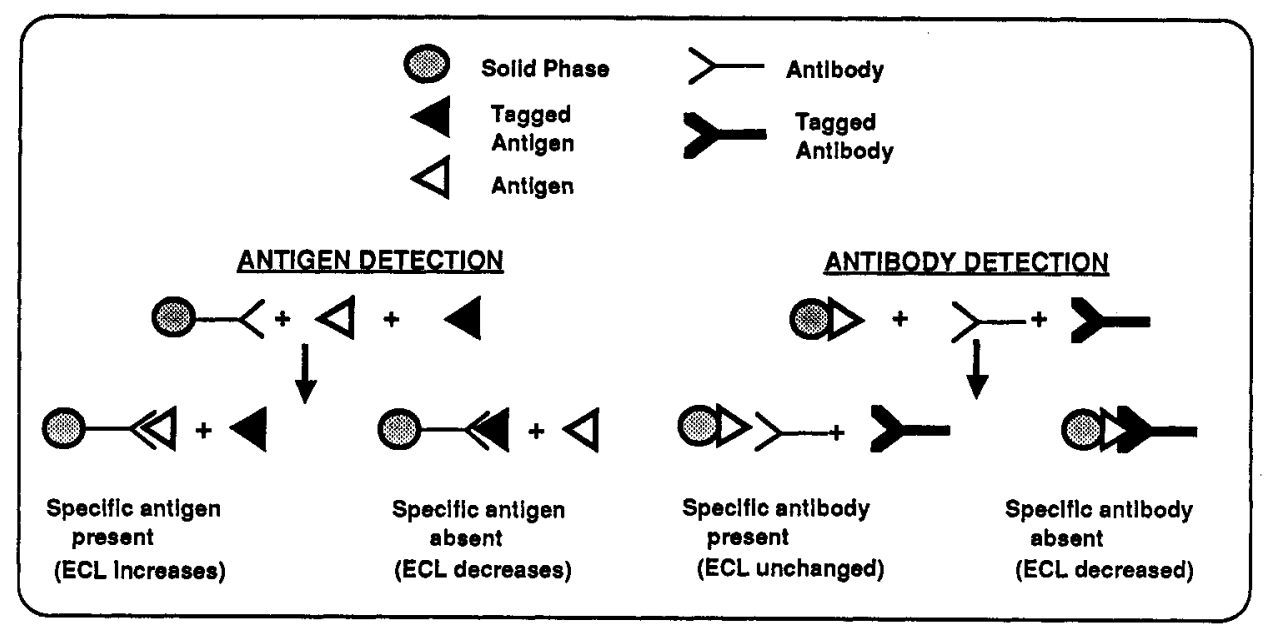

Fig. 15

\section{CONCLUSIONS}

Although electrochemistry is often considered to be an old and mature field, it remains an important one because of its central role in many areas that address societal needs. Moreover, the incorporation of the many recent advances in technology and other areas of science has continued to make electrochemistry and electroanalysis active and vital areas of research. This lecture has only touched on some of these new research topics, certainly with an overemphasis on results from our own laboratory. However it is clear that electrochemistry will continue to play an important role in responding to the challenges to science in the 21 st century.

\section{REFERENCES}

1. G. Whitesides, Angew, Chem. Int.Ed.Engl, 29, 1209-1218 (1990).

2. A. J. Bard, K. Itaya, R. E. Malpas, and T. Teherani, J.Phys, Chem, 84, 1262-1266 (1980).

3. R. M. Wightman and D. O. Wipf in A. J. Bard, Electroanalytical Chemistry, Marcel Dekker, Inc., New York; Volume 15, pp. 267-353 (1988).

4. E. Garcia and A. J. Bard, J.Electrochem. Soc, 137, 2752-2759 (1990).

5. D. O. Wipf, E. W. Kristensen, M. R. Deakin, and R. M. Wightman, Anal Chem, 60, 306-310(1988),

6. C. A. Amatore, A. Jutland, and F. Pfluger, L.Electroanal. Chem., 218, 361-365 (1987).

7. C. $X u$ and L. R. Faulkner, private communication.

8. G. Binnig and H. Rohrer, Helv. Phys. Acta, 55, 726-735 (1982).

9. A. J. Bard and F-R. Fan in D. A. Bonnell, Scanning Tunneling Microscopy: Theory Techniques, and Aoplications, VCH Publ., New York; (1991) (In Press).

10. S. A. Hendricks, Y.T. Kim, and A. J. Bard (In Preparation).

11. H. Chang and A. J. Bard, J.Am. Chem. Sec., 113, 5588-5596 (1991).

12. C. E. D. Chidsey, D. N. Loiacono, T. Sleator, S. Nakahara, Surf. Sci, 200, 45 (1988).

13. R. L. MoCarley and A. J. Bard (Submitted).

14. Y-T. Kim, R. L. McCarley, and A. J. Bard (In Preparation).

15. S-L. Yau, C. M. Vitus, and B. C. Schardt, J.Am. Chem. Soc. 112, 3677-3679 (1990).

16. C. A. Widrig, A. Alves, and M. D. Porter, J.Am. Chem. Soc., 113, 2805-2806 (1991).

17. S. Manne, P. K. Hansma, J. Massie, V. B. Elings, A. A. Gewirth, Science, 251, 183-186 (1991).

18. A. J. Bard, F.-R. F. Fan, J. Kwak, and O. Lev, Anal Chem, 61, 132-138 (1989).

19. D. O. Wipf and A. J. Bard, J. Electrochem. Soc., 138, 469-474 (1991).

20. C. Lee, C. J. Miller, and A. J. Bard, Anal. Chem, 63, 78-83 (1991).

21. O. E. Hüsser, D. H. Craston, and A. J. Bard, J_Vac. Sci. Technol. B, 6, 1873-1876 (1988).

22. S. H. Jenkins, H. B. Halsall, and W. R. Heineman, Third Chemical Congress of North America, Toronto, June, 1988.

23. P. R. Unwin and A. J. Bard, (Submitted).

24. T. Abe, Y. Y. Lau, and A. G. Ewing, J.Am. Chem. Soc. (In Press).

25. J. T. MCDevitt, S. Ching, M. Sullivan, and R. W. Murray, J.Am. Chem. Soc, 111, 4528-4529 (1989).

26. C. R. Cabrera and A. J. Bard, J_Electroanal, Chem, 273, 147-160 (1989).

27. M. T. Carter, M. Rodríguez, and A. J. Bard, J.Am. Chem. Soc., 111, $8901-8911$ (1989).

28. M. Rodriguez, T. Kodadek, M. Torres, and A. J. Bard, Bioconiugate Chem. 1, 123-131 (1990).

29. L. R. Faulkner and A. J. Bard in A. J. Bard, Electroanalytical Chemistry, Marcel Dekker, Inc., New York; Volume 10, pp. 1-95 (1977).

30. I. Rubinstein and A. J. Bard, J.Am. Chem Sec, 103, 512-516 (1981).

31. J. B. Noffsinger and N. D. Danielson, Anal Chem, 59, 865-868 (1987).

32. D. Ege, W. G. Becker, and A. J. Bard, Anal. Chem, 56, 2413-2417 (1984).

33. J. K. Leland and M. J. Powell, J.Electrochem. Soc, 137, 257-263 (1990).

34. IGEN, Inc., 1530 E. Jefferson Street, Rockville, MD 20852. 